\title{
ON THE EFFECTIVENESS OF THE SCHRÖDER-BERNSTEIN THEOREM
}

\author{
J. B. REMMEL ${ }^{1}$
}

\begin{abstract}
The effectiveness of the classical equivalence theorem of Schröder and Bernstein is investigated using the tools of recursion theory. We prove one result which generalizes all the effective versions of the Schröder-Bernstein theorem which occur in the literature. In contrast, we show that Banach's strengthening of the Schröder-Bernstein theorem fails to be effective.
\end{abstract}

\section{Introduction.}

TheOREM I (SCHRÖDER-BERnSTEIN). Let $A$ and $B$ be sets and let $f: A \rightarrow B$ and $g$ : $B \rightarrow A$ be $1: 1$ functions, then there exists $a 1: 1$ function $h$ mapping $A$ onto $B$.

The first satisfactory proof of Theorem I was due to Felix Bernstein and was published in a book by Borel [2] in 1898. Schröder had announced the theorem in 1896 but his proof of it, also published in 1898, contained a flaw (see Korselt [5]). Cantor also gave a proof of the theorem in 1897 [3] which is why the result is sometimes referred to as the Cantor-Bernstein theorem; however Cantor's proof used the axiom of choice, which is unnecessary. In 1924, Banach [1] published a strenghening of the result which states that the function $h$ in Theorem I can be chosen so that $h \subseteq f \cup g^{-1}$.

THEOREM II (BANACH). Given $A, B, f$, and $g$ as in Theorem $I$, there exist partitions $A=A_{1} \cup A_{2}$ and $B=B_{1} \cup B_{2}$ such that $f \uparrow A_{1}$ ( $f$ restricted to $\left.A_{1}\right)$ maps $A_{1}$ onto $B_{1}$ and $\mathrm{g}^{-1} \uparrow A_{2}$ maps $A_{2}$ onto $B_{2}$.

In this paper we shall apply the basic ideas and techniques of recursion theory to study the effective content of Theorems I and II. We shall show that there are natural settings in which the Schröder-Bernstein theorem is effective. In fact, in $\$ 2$ we shall prove one theorem which at once generalizes all the known effective versions of Theorems I. In contrast, we shall show that Banach's theorem fails to be effective in all such settings, reflecting the fact that the choices one must make in all the usual proofs of Theorem II are in an essential way noneffective.

The recursion theory we assume can be found in [9]. Let $\varphi_{0}, \varphi_{1}, \ldots$ be an effective list of all partial recursive functions. We think of $\varphi_{i}$ as being computed by the $i$ th Turing machine and write $\varphi_{i}^{s}(x) \downarrow$ if the $i$ th Turing machine, when started on a tape coding $x$, gives an output in $s$ or fewer steps. We write $\varphi_{i}(x) \downarrow$ if $\exists s\left(\varphi_{i}^{s}(x) \downarrow\right)$.

Received by the editors May 27, 1980.

AMS (MOS) subject classifications (1970). Primary 02F99; Secondary 02F40, 04 A05.

${ }^{1}$ Partially supported by NSF grant MCS79-03406. 
Given subsets $A$ and $B$ of the natural numbers $N$, we write $A \leqslant T$ if $A$ is Turing reducible to $B$ and $A \equiv_{T} B$ if $A \leqslant T B$ and $B \leqslant T A$. The (Turing) degree of $A$, $\operatorname{deg}(A)$, is $\left\{B \subseteq N \mid B \equiv_{T} A\right\} .\langle\rangle:, N \times N \rightarrow N$ will denote a fixed $1: 1$ onto recursive pairing function. Given a partial function $h: A \rightarrow N$ where $A \subseteq N$, we let $\operatorname{deg}(h)=\operatorname{deg}(\{\langle x, h(x)\rangle: x \in A\})$. $0^{\prime}$ will denote the highest possible Turing degree of any recursively enumerable set. If $f$ is a function, $f^{k}$ will denote $f$ composed with itself $k$ times, and $\operatorname{dom} f$ and $\operatorname{ran} f$ will denote the domain and range of $f$, respectively. Given a set $D \subseteq N$, let $\chi_{D}(x)$ equal 1 if $x \in D$ and 0 otherwise. Given a finite set $\left\{x_{1}<\cdots<x_{n}\right\}$, we call $2^{x_{1}}+\cdots+2^{x_{n}}$ its canonical index and let 0 be the canonical index of $\varnothing$. $D_{x}$ will denote the finite set with canonical index $x$.

1. The most natural thing to do to give an effective version of Theorem $I$ is to assume that the sets $A$ and $B$ are recursive, the functions $f$ and $g$ are partial recursive, and require the function $h$ to be partial recursive. In this case, Theorem I is trivially effectively since we can effectively list, in order of magnitude, $A$ as $a_{0}, a_{1}, \ldots$ and $B$ as $b_{0}, b_{1}, \ldots$, and then automatically the function $h: A \rightarrow B$ where $h\left(a_{i}\right)=b_{i}$ for all $i$ will be a $1: 1$ partial recursive function mapping $A$ onto $b$. However, even in this simple setting, Theorem II fails to be effective in quite a strong way.

Theorem 1. Assume $A$ and $B$ are infinite recursive sets. Then there exist $1: 1$ partial recursive functions $f: A \rightarrow B$ and $g: B \rightarrow A$ such that $\operatorname{ran} f$ and $\operatorname{ran} g$ are recursive and yet there is no partial recursive function $h \subseteq f \cup g^{-1}$ such that $h$ maps A $1: 1$ onto $B$.

Proof. Before constructing the functions $f$ and $g$, it will be useful to review the proof of Banach's theorem. Given $f$ and $g$ as in Theorem II, we introduce an equivalence relation on $A$. Given $x, y \in A$, we write $x \sim y$ if either $y \in$ $\left\{x, g \circ f(x),(g \circ f)^{2}(x), \ldots\right\}$ or $x \in\left\{y, g \circ f(y),(g \circ f)^{2}(y), \ldots\right\}$. The equivalence classes of $A$ under $\sim$ and the corresponding images under $g^{-1}$ can be classified as one of 4 types pictured below.

Type 1. Cycles

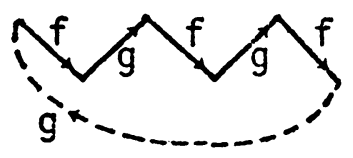

Type 2. Two way infinite chain

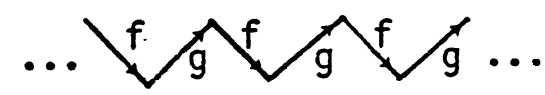

Type 3. One way infinite chain with initial element not in $\operatorname{ran} g$

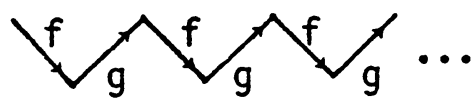


Type 4. One way infinite chain with initial element not in $\operatorname{ran} f$

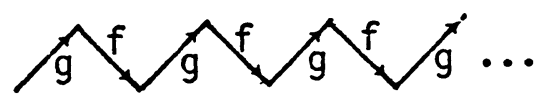

It is easy to see that equivalence classes of Types 1 or 2 can be either subsets of $A_{1}$ or $A_{2}$ in the partition, but the equivalence classes of Type 3 must be subsets of $A_{1}$ while equivalence classes of Type 4 must be subsets of $A_{2}$.

The basic idea of our proof is to construct $f$ and $g$ in stages, so that only equivalence classes of Type 2, 3, or 4 occur, and control the equivalence classes in such a way so as to ensure no partial recursive function can satisfy the conclusion of Banach's theorem. First, we partition $A=X_{0} \cup X_{1} \cup \cdots$ and $B=Y_{0} \cup Y_{1}$ $\cup \cdots$ into infinite sequences of pairwise disjoint infinite recursive sets. For each $i, X_{i}$ will be an equivalence class and $Y_{i}$ will be $g^{-1}\left(X_{i}\right)$ and we will use $X_{i}$ and $Y_{i}$ to ensure that the $i$ th partial recursive function $\varphi_{i}$ is not both contained in $f \cup g^{-1}$ and a mapping of $A 1: 1$ onto $B$. To accomplish this for each $i$, it is easy to see that we need only construct $f$ and $g$ so that $X_{i}$ is an equivalence class of Type 4 if $\varphi_{i}$ agrees with $f$ on $X_{i}$ while $X_{i}$ is an equivalence class of Type 3 if $\varphi_{i}$ agrees with $g^{-1}$ on $X_{i}$.

Fix $i$, then at stage 0 of our definition of $f$ on $X_{i}$ and $g$ on $Y_{i}$, let $x_{0}$ and $y_{0}$ be the least elements of $X_{i}$ and $Y_{i}$, respectively, and let $f\left(x_{0}\right)=y_{0}$. At stage $s+1$, we will be in one of two cases. First, if $\varphi_{i}^{s+1}\left(x_{0}\right)$ is not defined, then at stage $s$, we will have specified $x_{-s}, \ldots, x_{0}, \ldots, x_{s}$ in $X_{i}$ and $y_{-s}, \ldots, y_{0}, \ldots, y_{s}$ in $Y_{i}$ and defined $f\left(x_{j}\right)=y_{j}$ for $-s \leqslant j \leqslant s$ and $g\left(y_{j}\right)=x_{j+1}$ for $-s \leqslant j<s$. Then at stage $s+1$, we will extend our sequences at both ends, that is, we let $x_{-s-1}<x_{s+1}$ be the least two elements of $X_{i}-\left\{x_{-s}, \ldots, x_{0}, \ldots, x_{s}\right\}$ and $y_{-s-1}<y_{s+1}$ be the least two elements of $Y_{i}-\left\{y_{-s}, \ldots, y_{0}, \ldots, y_{s}\right\}$ and define $f\left(x_{-s-1}\right)=y_{-s-1}, f\left(x_{s+1}\right)=y_{s+1}$, $g\left(y_{-s-1}\right)=x_{-s}$, and $g\left(y_{s}\right)=x_{s+1}$. But if $\varphi_{i}^{s+1}\left(x_{0}\right) \downarrow$, then let $t$ be the least stage such that $\varphi_{i}^{t+1}\left(x_{0}\right) \downarrow$. Thus at stage $t$, we have two sequences $x_{-t}, \ldots, x_{0}, \ldots, x_{t}$ in $X_{i}$ and $y_{-t}, \ldots, y_{0}, \ldots, y_{t}$ in $Y_{i}$ as above. Now if $\varphi_{i}\left(x_{0}\right) \neq f\left(x_{0}\right)=y_{0}$, then at stage $t+1$ we will extend our sequences at both ends as described above, but at all later stages $s$, we will only extend our sequences at the positive end so that our sequences will be of the form $x_{-t-1}, \ldots, x_{0}, \ldots, x_{s}$ and $y_{-t-1}, \ldots, y_{0}, \ldots, y_{s}$. Note that in this case, $x_{-t-1}$ will not be in ran $g$ so that the sequence will be of Type 4. If $\varphi_{i}\left(x_{0}\right)=f\left(x_{0}\right)$, then at stage $t+1$, we will choose $x_{t+1}$ to be the least element of $X_{i}-\left\{x_{-t}, \ldots, x_{0}, \ldots, x_{t}\right\}$ and $y_{-t-1}<y_{t+1}$ to be the least two elements of $Y_{i}-\left\{y_{-t}, \ldots, y_{0}, \ldots, y_{t}\right\}$ and define $g\left(y_{-t-1}\right)=x_{-t}, g\left(y_{t}\right)=x_{t+1}$, and $f\left(x_{t+1}\right)=y_{t+1}$. Then at all later stages $s$, we will extend our sequences only at the positive end so that our sequences will be of the form $x_{-t}, \ldots, x_{0}, \ldots, x_{s}$ and $y_{-t-1}, \ldots, y_{0}, \ldots, y_{s}$. In this case, $y_{-t-1}$ will not be in $\operatorname{ran} f$ so that the sequence will be of Type 3 .

This completes our description of $f$ and $g$. It is easy to see that our construction is completely effective so that $f: A \rightarrow B$ and $g: B \rightarrow A$ will be $1: 1$ partial recursive functions. For each $i$, we will be in one of three possible cases at the end of our construction. (1) $\varphi_{i}\left(x_{0}\right)$ is not defined, in which case $X_{i}$ is an equivalence class of Type 2; (2) $\varphi_{i}\left(x_{0}\right) \downarrow$ and $\varphi_{i}\left(x_{0}\right) \neq f\left(x_{0}\right)$, in which case $X_{i}$ is an equivalence class of Type 3; or (3) $\varphi_{i}\left(x_{0}\right) \downarrow$ and $\varphi_{i}\left(x_{0}\right)=f\left(x_{0}\right)$, in which case $X_{i}$ is an equivalence class of 
Type 4. It now follows by our previous remarks that there is no partial recursive $h \subseteq f \cup g^{-1}$ mapping $A 1: 1$ onto $B$. Finally, it is easy to check that our construction ensures that $\operatorname{ran} f, B-\operatorname{ran} f, \operatorname{ran} g$, and $A-\operatorname{ran} g$ are all recursively enumerable and hence they are all recursive since $A$ and $B$ are recursive.

We remark that since for infinitely many $i, \varphi_{i}$ is totally undefined, there will be infinitely many equivalence classes of Type 2 for the $f$ and $g$ constructed in Theorem 1 and thus there are $2^{\kappa_{0}}$ functions $h \subseteq f \cup g^{-1}$ such that $h$ maps $A$ 1:1 onto $B$. This is necessarily the case since if $f: A \rightarrow B$ and $g: B \rightarrow A$ are $1: 1$ partial recursive functions where $A, B, \operatorname{ran} f$, and $\operatorname{ran} g$ are recursive, and there are only finitely many $h \subseteq f \cup g^{-1}$ which map $A 1: 1$ onto $B$, i.e., there are only finitely many equivalence classes of Types 1 and 2 , then it is not difficult to see that all such $h$ are partial recursive. (This result could be regarded as an effective version of Banach's theorem.) Nevertheless, if one is willing to drop the requirements that ran $f$ and ran $g$ are recursive in Theorem 1, then we can still construct recursive counterexamples to Banach's theorem in the case where there is a unqiue $h \subseteq f \cup$ $g^{-1}$ which maps $A$ 1:1 onto $B$. We say $E$ is a recursive limit of finite sets $E_{0}, E_{1}, \ldots$ if there is a recursive function $\varphi_{e}$ such that for each $i, E_{i}=D_{\varphi_{e}(i)}$ and $\chi_{E}=\lim _{s} \chi_{E_{s}}$.

THEOREM 2. Let $A$ and $B$ be infinite recursive sets. Then for any $E$ which is a recursive limit of finite sets $E_{0}, E_{1}, \ldots$, there exist $1: 1$ partial recursive functions $f$ : $A \rightarrow B$ and $g: B \rightarrow A$ such that there is $a$ unique $h \subseteq f \cup g^{-1}$ mapping $A 1: 1$ onto $B$ and yet $\operatorname{deg}(h)=\operatorname{deg}(E)$.

Proof. Note that if $h$ is partial recursive with a recursive domain, then $\operatorname{deg}(h)$ is the degree of the recursive sets, hence $h$ is partial recursive iff $E$ is recursive. Since not all such $E$ are recursive, it follows that $h$, in general, will not be partial recursive.

We will construct $f$ and $g$ much as in Theorem 1, only this time we use the equivalence classes to code $E$ into $h$. So assume the notation of Theorem 1. For each $i$, let stage 0 of the definition of $f$ on $X_{i}$ and $g$ on $Y_{i}$ be as before. Assume at stage $s \geqslant 0$, we have defined $x_{-k_{s}}, \ldots, x_{0}, \ldots, x_{s}$ in $X_{i}$ and $y_{-j}, \ldots, y_{0}, \ldots, y_{s}$ in $Y_{i}$ such that $j_{s}=k_{s}$ or $k_{s}+1$ and $f\left(x_{n}\right)=y_{n}$ for $-k_{s} \leqslant n \leqslant s$ and $g\left(y_{n}\right)=x_{n+1}$ for $-j_{s} \leqslant n<s$. If $s>0$, assume further that either (a) $i \in E_{s}$ and $j_{s}=k_{s}$ so that we have an initial segment of Type 3 , or (b) $i \notin E_{s}$ and $j_{s}=k_{s}+1$ so that we have an initial segment of Type 4. Then at stage $s+1$, if either $i$ is in both or out of both $E_{s}$ and $E_{s+1}$ extend the sequences on the positive side as in Theorem 1. If $i \in E_{s}$ but $i \notin E_{s+1}$, then let $k_{s+1}=k_{s}, j_{s+1}=j_{s}+1$, and let $y_{-j_{s+1}}<y_{s+1}$ be the least two elements of $Y_{i}-\left\{y_{-j_{s}}, \ldots, y_{0}, \ldots, y_{s}\right\}$ and $x_{s+1}$ be the least element of $X_{i}-$ $\left\{x_{-k_{s}}, \ldots, x_{0}, \ldots, x_{s}\right\}$ and define $g\left(y_{-j_{s+1}}\right)=x_{-j_{s}}, g\left(y_{s}\right)=x_{s+1}$, and $f\left(x_{s+1}\right)=$ $y_{s+1}$. If $i \notin E_{s}$ but $i \in E_{s+1}$, let $k_{s+1}=k_{s}+1, j_{s+1}=j_{s}$ and let $x_{-k_{s+1}}<x_{s+1}$ be the least two elements of $X_{i}-\left\{x_{-k_{s}}, \ldots, x_{0}, \ldots, x_{s}\right\}$ and $y_{s+1}$ be the least element of $Y_{i}-\left\{y_{-j_{s}}, \ldots, y_{0}, \ldots, y_{s}\right\}$ and define $f\left(x_{-k_{s+1}}\right)=y_{-j}, g\left(y_{s}\right)=x_{s+1}$, and $f\left(x_{s+1}\right)=y_{s+1}$. 
As in Theorem 1, our construction ensures that $f: A \rightarrow B$ and $g: B \rightarrow A$ are $1: 1$ partial recursive functions. Moreover, we have ensured that for each $i$, either (a) $i \in E$ and $X_{i}$ is an equivalence class of Type 3 or (b) $i \notin E$ and $X_{i}$ is an equivalence class of Type 4. Thus there is a unique $h \subseteq f \cup g^{-1}$ such that $h$ maps $A \quad 1: 1$ onto $B$ and $h \uparrow X_{i}=f \uparrow X_{i}$ iff $i \in E$. It then follows that $\operatorname{deg}(h)=$ $\operatorname{deg}(E)$.

It is not difficult to see that if $A$ and $B$ are recursive sets and $f: A \rightarrow B$ and $g$ : $B \rightarrow A$ are 1:1 partial recursive functions for which there are only finitely many $h \subseteq f \cup g^{-1}$ mapping $A 1: 1$ onto $B$, then $\operatorname{deg}(h)$ is recursive in $0^{\prime}$. By the Shoenfield Limit Lemma [10], a set $E$ is recursive in $0^{\prime}$ iff $E$ is a recursive limit of finite sets. Thus Theorem 2 is the best possible. We note that using a slight modification of the construction of Theorem 2, we can diagonalize over all possible recursive limits of finite sets to produce for any infinite recursive sets $A$ and $B, 1: 1$ partial recursive functions $f: A \rightarrow B$ and $g: B \rightarrow A$ such that there is no $h \subseteq f \cup$ $g^{-1}$ mapping $A$ 1: 1 onto $B$ with $h$ recursive in $0^{\prime}$. We shall, however, not give the details.

We end this section with an interesting version of Banach's theorem in the setting of bipartite graphs given by Mirsky and Perfect in [7] which will show that our counterexamples in Theorems 1 and 2 have interesting graph theoretic interpretations. A bipartite graph is a triple $\langle A, B, E\rangle$ where $E$ is a set of unordered pairs $\{x, y\}$ with $x \in A$ and $y \in B$. Elements of $A$ and $B$ are called nodes and elements of $E$ are called edges. If $e=\{x, y\} \in E$, we say $x$ and $y$ meet $e$. A matching $M$ is a set of edges so that each node meets at most one edge in $M$. The following theorem is then easily seen to be equivalent to Theorem II.

THEOREM 3. Let $\langle A, B, E\rangle$ be a bipartite graph such that there are matchings $M_{1}$ and $M_{2}$ so that every node of $A$ meets an edge of $M_{1}$ and every node of $B$ meets an edge of $M_{2}$. Then there is a matching $M$ such that every node in $A$ or $B$ meets an edge in $M$.

We note that when put in this graph theoretic context, our counterexamples can be seen to be related to the work of Manaster and Rosenstein [6].

2. There are two other effective versions of the Schröder-Bernstein theorem in the literature. In both effective versions of Theorem I, $A$ and $B$ are assumed merely to be subsets of $N, f$ and $g$ are the restrictions of $1: 1$ partial recursive functions, and we conclude that $h$ is the restriction of a $1: 1$ partial recursive function. However, both versions require some additional hypothesis because of the following counterexample. Let $E$ and $\theta$ denote the even and odd numbers, respectively, and let $k: N \rightarrow E$ be the recursive function defined by $k(e)=2 e$ for all $e$. Now if $A=E$ and $B=A \cup C$ where $C \subseteq \mathcal{O}$, then $f: A \rightarrow B$ and $g: B \rightarrow A$ where $f=k \uparrow A$ and $g=k \uparrow B$ are the restrictions of $1: 1$ partial recursive functions. The existence of the restriction of a $1: 1$ partial recursive function $h$ mapping $A$ onto $B$ would imply $C$ is r.e. Thus, choosing $C$ to be a non-r.e. subset of $\theta$ shows $h$ cannot exist in general. To state the extra hypothesis which we need, we must introduce the notions of $1: 1$ equivalence due to Myhill [8] and recursive equivalence types 
due to Dekker and Myhill [4]. Given $A, B \subseteq N$, we say $A$ is $1: 1$ reducible to $B$ (via $f), A \leqslant_{1} B$, if there is a total $1: 1$ recursive function $f: N \rightarrow N$ such that $\forall x \in N(x$ $\in A$ iff $f(x) \in B$ ). The first of our effective versions of Theorem $I$ is due to Myhill [8].

THEOREM 4. If $A \leqslant 1 B$ and $B \leqslant 1$, then there exists a recursive function $h$ mapping $N 1: 1$ onto $N$ such that $h(A)=B$.

Given $A, B \subseteq N$, we say $A$ is recursively equivalent to $B, A \sim_{r} B$, if there exists a 1:1 partial recursive function $p$ such that $\operatorname{dom} p \supseteq A$ and $p\lceil A$ maps $A$ onto $B$. $\langle A\rangle=\left\{B \subseteq N \mid A \sim_{r} B\right\}$ is called the recursive equivalence type or R.E.T. of $A$. $\langle A\rangle$ can be viewed as the effective cardinality of $A$. We define $A \leqslant r B$ if $A$ is recursively equivalent to an r.e. separated subset of $B$, i.e., if there are disjoint r.e. set $W_{1}$ and $W_{2}$ such that $W_{1} \cup W_{2} \supseteq B$ and $A \sim_{r} B \cap W_{1}$. Given R.E.T.s $\alpha$ and $\beta$, we define $\alpha \leqslant_{r} \beta$ if there exist $A \in \alpha$ and $B \in \beta$ with $A \leqslant r B$. The important result that $\leqslant r$ partially orders the R.E.T.s follows from the next theorem due to Dekker and Myhill [4] which is yet another effective version of the Schröder-Bernstein theorem.

THeOREM 5. If $A \leqslant r B$ and $B \leqslant r A$, then $A \sim_{r} B$.

Theorems 4 and 5 are different since it is easy to construct counterexamples to show that $\leqslant_{1}$ and $\leqslant_{r}$ do not coincide. Our next result will yield Theorems 4 and 5 as well as the trivial effective version of Theorem I mentioned in $\$ 1$ as corollaries. We wish to acknowledge that one of the basic ideas for the proof of Theorem 6 to follow comes from an unpublished proof of Theorem 5 due to A. Manaster.

THEOREM 6. Assume $A, B \subseteq N$ and $f$ and $g$ are $1: 1$ partial recursive functions where $\operatorname{dom} f \supseteq A$, dom $g \supseteq B$, and there exist r.e. sets $W_{1}, W_{2}, U_{1}$, and $U_{2}$ such that (i) $W_{1} \cap W_{2}=\varnothing=U_{1} \cap U_{2}$, (ii) $f(A) \subseteq W_{1} \cap A$ and $f(\operatorname{dom} f-A) \cap B \subseteq$ $W_{2}$, and (iii) $g(B) \subseteq U_{1} \cap A$ and $g(\operatorname{dom} g-B) \cap A \subseteq U_{2}$. Then there exists $a 1: 1$ partial recursive function $h$ such that $A \subseteq \operatorname{dom} h$ and $h \uparrow A$ maps $A$ onto $B$.

Proof. First we claim, we can without loss of generality assume $\operatorname{dom} f \operatorname{ran} g, U_{1}$ and $U_{2}$ are subsets of the even numbers $E$ and $\operatorname{dom} g, \operatorname{ran} f, W_{1}$, and $W_{2}$ are subsets of the odd numbers $\theta$. For if we are not in such a situation, let $k: N \rightarrow E$ be defined by $k(e)=2 e$ for all $e$ and $l: N \rightarrow \theta$ be defined by $l(e)=2 e+1$ and then let $A^{\prime}=k(A), U_{1}^{\prime}=k\left(U_{1}\right), U_{2}^{\prime}=k\left(U_{2}\right), B^{\prime}=l(B), W_{1}^{\prime}=l\left(W_{1}\right), W_{2}^{\prime}=l\left(W_{2}\right)$, $f^{\prime}=l \circ f \circ k^{-1}$, and $g^{\prime}=k \circ g \circ l^{-1}$. Our argument will show there is $1: 1$ recursive function $h^{\prime}$ with $\operatorname{dom} h^{\prime} \subseteq E$ and $\operatorname{ran} h^{\prime} \subseteq \vartheta$ such that $h^{\prime} \uparrow A^{\prime}$ maps $A^{\prime}$ onto $B^{\prime}$. Then $h=l^{-1} \circ h^{\prime} \circ k$ will be a $1: 1$ partial recursive function required by the theorem.

So assume $\operatorname{dom} f, \operatorname{ran} g, U_{1}, U_{2} \subseteq E$ and $\operatorname{dom} g, \operatorname{ran} f, W_{1}$, and $W_{2} \subseteq \mathcal{\theta}$. We shall build $h$ in stages $s$. For any $s$, let $f^{s}=\left\{(x, f(x)) \mid x \leqslant s \& f^{s}(x) \downarrow\right\}$ and $g^{s}=\left\{(x, g(x)) \mid x \leqslant s \& g^{s}(x) \downarrow\right\}$. At stage $s$, we call a sequence of distinct elements $\left\langle a_{0}, a_{1}, \ldots, a_{n}\right\rangle$ a chain if either: (i) $a_{0} \in \operatorname{dom} f^{s},\left\langle a_{0}, \ldots, a_{n}\right\rangle$ is of the form $\left\langle a_{0}\right.$, $\left.f^{s}\left(a_{0}\right), g^{s} \circ f^{s}\left(a_{0}\right), f^{s} \circ g^{s} \circ f^{s}\left(a_{0}\right), \ldots\right\rangle, a_{0}, a_{2}, a_{4}, \ldots$ are in $U_{1}$, and $a_{1}, a_{3}, \ldots$ 
are in $W_{1}$; or (ii) $a_{0} \in \operatorname{dom} g^{s},\left\langle a_{0}, \ldots, a_{n}\right\rangle$ is of the form $\left\langle a_{0}, g^{s}\left(a_{0}\right), f^{s} \circ g^{s}\left(a_{0}\right)\right.$, $\left.g^{s} \circ f^{s} \circ g^{s}\left(a_{0}\right), \ldots\right\rangle, a_{0}, a_{2}, \ldots$ are in $W_{1}$, and $a_{1}, a_{3}, \ldots$ are in $U_{1}$. We call a chain $\left\langle a_{0}, \ldots, a_{n}\right\rangle$ closed at stage $s$ if either $a_{0} \in \operatorname{dom} f^{s}$ and $g^{s^{-1}}\left(a_{0}\right)=a_{n}$ or $a_{0} \in \operatorname{dom} g^{s}$ and $f^{s^{-1}}\left(a_{0}\right)=a_{n}$. We call a chain $C=\left\langle a_{0}, \ldots, a_{n}\right\rangle$ maximal at stage $s$ if either $C$ is closed and $a_{0}$ is the minimum element of $C$ or $C$ is not closed and $C$ is not a proper subsequence of any chain at stage $s$. Let $C_{1}^{s}, \ldots, C_{n_{3}}^{s}$ denote the maximal chains at stage $s$. We note that our assumptions on $W_{1}, W_{2}, U_{1}$, and $U_{2}$ ensure that for any maximal chain $C_{i}^{s}$ either (a) $C_{i}^{s} \cap E$ is contained in $A$ and $C_{i}^{s} \cap \theta$ is contained in $B$ or (b) $C_{i}^{s} \cap E \cap A=\varnothing$ and $C_{i}^{s} \cap \theta \cap B=\varnothing$. We will define a finite function $h^{s}$ at stage $s$ so that if $h^{s}(x)=y$, then $x$ and $y$ lie in the same maximal chain. Moreover, we will ensure that for all $s, h^{s} \subseteq h^{s+1}$. We say $x \in N$ is free at stage $s$ if either $x \in E-\operatorname{dom} h^{s}$ or $x \in \mathcal{O}-\operatorname{ran} h^{s} . h^{s}$ will be defined so that for any maximal chain $C_{i}^{s}$ which is not closed, both $C_{i}^{s} \cap E$ and $C_{i}^{s} \cap \mathcal{O}$ contain at least one free element. This given, our instructions at stage $s+1$ are very simple. One simply considers the set of maximal chains at stage $s+1, C_{1}^{s+1}, \ldots, C_{n_{i+1}}^{s+1}$. For any chain $C_{i}^{s+1}$ which is either closed and $C_{i}^{s+1} \cap E$ and $C_{i}^{s+1} \cap \mathcal{O}$ contain free elements or is such that both $C_{i}^{s+1} \cap E$ and $C_{i}^{s+1} \cap \mathcal{O}$ contain at least two free elements at stage $s$, we take the least free member of $C_{1}^{s+1} \cap E, x$, and the least free member of $C_{i}^{s+1} \cap \theta, y$, and define $h^{s+1}(x)=y$. We let $h=\cup_{s} h^{s}$ so that automatically $h$ is a $1: 1$ partial recursive function since our procedure is completely effective.

It easily follows from our definitions that any maximal chain $C_{i}^{s}$ at stage $s$ is contained in a maximal chain $C_{j}^{s+1}$ at stage $s+1$. Thus for any $C_{i}^{s}$ there will be a unique sequence $C_{i}^{s}=C_{i_{s}}^{s} \subseteq C_{i_{s+1}}^{s+1} \subseteq C_{i_{s+2}}^{s+2} \subseteq \cdots$ so we let $C=\cup_{t>s} C_{i_{\text {s }}}^{s}$. By our remarks earlier, either $C \subseteq A \cup B$ or $C \cap(A \cup B)=\varnothing$. In case $C \subseteq A \cup B, C$ can be pictured as one of the Types 1-4 of Theorem 1 and it is easy to check that our choice having $h$ send the least free element of $E \cap C_{j}^{s+1}$ to least free element of $\theta \cap C_{j}^{s+1}$ will ensure that $h$ maps $E \cap C 1: 1$ onto $\theta \cap C$. It then follows that $h$ maps $A 1: 1$ onto $B$.

Finally we should remark how the three effective versions of Theorem I all are special cases of this theorem. For Theorem 4, we have total $1: 1$ recursive $f$ and $g$ such that $f(A) \subseteq B, f(N-A) \subseteq N-B, g(B) \subseteq A$ and $g(N-B) \subseteq N-A$. We can then take $U_{1}=W_{1}=N$ and $U_{2}=W_{2}=\varnothing$. In this case, it is not difficult to check that the $h$ constructed above will be a total recursive function which maps $N$ onto $N$. Note in Theorem 5 , we have a much stronger kind of separation of the range of $f$ and $g$ within $A$ and $B$, respectively, than we require in Theorem 6. That is, in Theorem 5, $f$ and $g$ are 1:1 partial recursive functions for which there are r.e. sets $M_{1}, M_{2}, N_{1}$ and $N_{2}$ such that $f(A) \cap B \subseteq M_{1}, B-f(A) \subseteq M_{2}, g(B) \cap A \subseteq$ $N_{1}$, and $A-g(B) \subseteq N_{2}$. Thus, clearly $U_{i}=M_{i}$ and $W_{i}=N_{i}$ for $i=1,2$ satisfy the hypothesis of Theorem 6. Finally in the trivial case where $A$ and $B$ are recursive, we can assume $\operatorname{dom} f=A$ and $\operatorname{dom} g=B$ so that we can let $W_{1}=U_{1}$ $=N$ and $W_{2}=U_{2}=\varnothing$.

We should note that all the counterexamples for Banach's theorem described in $\$ 1$ satisfy the hypothesis of Theorem 5 . In fact, our first counterexample given in 
Theorem 1 shows Banach's theorem fails, even under the strongest possible type of separation conditions for $\operatorname{ran} f$ in $B$ and $\operatorname{ran} g$ in $A$.

\section{REFERENCES}

1. S. Banach, Un théorème sur les transformations biunivoques, Fund. Math. 6 (1924), 236-239.

2. E. Borel, Leçons sur la théorie des fonctions, Paris, 1898.

3. G. Cantor, Beiträge zur Begründung der transfiniten Mengenlehre. II, Math. Ann. 49 (1897), 207-246.

4. J. C. E. Dekker and J. Myhill, Recursive equivalence types (Monograph), Univ. California Publ. Math. 3 (1960).

5. A. Korselt, Über einen Beweis des Äquivalenzsatzes, Math. Ann. 70 (1911), 294-296.

6. A. Manaster and J. Rosenstein, Effective matchmaking (recursion theoretic aspects of a theorem of Philip Hall), Proc. London Math. Soc. 25 (1972), 615-654.

7. L. Mirsky and H. Perfect, Systems of representatives, J. Math. Anal. Appl. 15 (1966), 520-568.

8. J. Myhill, Creative sets, Z. Math. Logik Grundlagen Math. 1 (1955), 97-108.

9. H. Rogers, Jr., Theory of recursive functions and effective computability, McGraw-Hill, New York, 1967.

10. J. Shoenfield, Degrees of unsolvability, North-Holland, New York, 1971.

Department of Mathematics, University of California at San Diego, la Jolla, California 92093 